\title{
Uniportal video-assisted thoracoscopic early learning curve for major lung resections in a high volume training center
}

\author{
Luis Angel Hernandez-Arenas ${ }^{1,2 \#}$, Lei Lin ${ }^{2 \#}$, Rushmi D. Purmessur ${ }^{1}$, Yiming Zhou ${ }^{2}$, Gening Jiang ${ }^{2}$, \\ Yuming $\mathrm{Zhu}^{2}$
}

${ }^{1}$ Department of Thoracic Surgery Birmingham Heartlands Hospital, NHS Trust Foundation, Birmingham, UK; ${ }^{2}$ Department of Thoracic Surgery, Shanghai Pulmonary Hospital, Tongji University School of Medicine, Shanghai 200433, China

Contributions: (I) Conception and design: LA Hernandez-Arenas, L Lin; (II) Administrative support: G Jiang, Y Zhu; (III) Provision of study materials or patients: L Lin, LA Hernandez-Arenas, Y Zhu; (IV) Collection and assembly of data: LA Hernandez-Arenas, L Lin, Y Zhu; (V) Data analysis and interpretation: RD Purmessur, Y Zhou; (VI) Manuscript writing: All authors; (VII) Final approval of manuscript: All authors.

\#These authors contributed equally to this work.

Correspondence to: Dr. Luis Angel Hernandez-Arenas. Department of Thoracic Surgery Birmingham Heartlands Hospital, NHS Foundation Trust, Bordesley Green E, Birmingham B9 5SS, UK. Email: luis.hernandez@nhs.net.

Background: Uniportal video-assisted thoracoscopic surgery (VATS) for major lung resections is a novel upcoming approach, with increasing popularity worldwide. However, there is little literature regarding this technique's learning curve. We present our experience of the early learning curve of the uniportal VATS major lung resections in a high volume training centre, whilst analysing the advantages.

Methods: Sixty selected consecutive patients underwent uniportal VATS major lung resections, for early stage disease of NSCLC and benign disease during the learning curve of a single surgeon in a high volume training centre from July to October 2015. The perioperative variables and outcomes were collected prospectively and analysed retrospectively.

Results: The 60 patients undergoing a uniportal VATS approach included 47 lobectomies and 13 segmental resections, among which 56 cases of lung cancer and 4 of benign pulmonary disease were noted. Right upper lobectomy (RUL) was the most common procedure (42\%). The mean operation time was $192.3 \pm 45.4$ minutes, average blood loss was $167.9 \pm 94.4 \mathrm{~mL}$. For patients with lung cancer, the total amount of lymph node stations sampled or dissected were $4.2 \pm 0.8$. Chest drain duration was $2.9 \pm 0.9$ days and length of hospital stay (LOS) was $4.38 \pm 1$ days. Prolonged air leak (PAL) was the most common complication in $8.3 \%$ of the cases. PAL was the cause of prolonged hospital stay. One case was converted to thoracotomy for major bleeding. There were no deaths 30 days after surgery or readmissions. All cases had a R0 complete cancer resection on histology.

Conclusions: The uniportal VATS lobectomy and segmentectomy early learning curve in a high volume training centre is a safe venture, allowing surgeons to reach an expert level faster and perform more complex resections with a shorter training time.

Keywords: Video-assisted thoracoscopic surgery (VATS); learning curve; uniportal

Submitted Feb 01, 2018. Accepted for publication Mar 20, 2018.

doi: $10.21037 /$ jtd.2018.04.16

View this article at: http://dx.doi.org/10.21037/jtd.2018.04.16 


\section{Introduction}

Uniportal video assisted-thoracic surgery (VATS) was first reported in a series of six cases by Yamamoto et al. (1) and this work was followed by Rocco and his group at the beginning of the year 2000. They proposed making a small enough incision to allow introduction of the instruments in the same wound parallel to the video thoracoscope. They initially performed and described the experience in minor pulmonary resections including wedge resections, pneumothorax, pleural and lung biopsies with good results (2-4).

The first major pulmonary resection by uniportal approach was described by Gonzalez-Rivas in 2011 (5). Since then the technique has evolved and demonstrated that it is safe, effective and reproducible $(6,7)$.

Increasingly complex surgeries such as segmentectomies, pneumonectomies, sleeve resections, angioplasties, carina resections and complex resections have been shown to be feasible by uniportal approach with similar results as by conventional approach (8-11).

Uniportal VATS pulmonary resections have emerged as a less invasive technique than the conventional multiport approach for the treatment of lung cancer and various groups have been increasingly trying the uniportal vats approach around the world (12). A recent meta-analysis compared VATS lobectomies performed via a uniportal approach and a multiport approach and revealed more favorable outcomes with a uniportal approach (13).

There is no randomized study to date proving that uniportal VATS is better than the conventional approach or even thoracotomy, although there are meta-analysis studies indicating that conventional VATS has better results than thoracotomy, and a meta-analysis comparing uniportal VATS versus conventional VATS demonstrating better results, thereby favouring the uniportal approach (13).

Moreover, there are no recommendations or guidelines that dictate when to use a uniportal, conventional VATS or open approach; this depends entirely on the experience of the surgeon. In 2012, the VATS consensus meeting, bringing together a panel of 55 experts, was carried out in Edinburgh, where the basis and certain recommendations for VATS were laid. The general consensus was that it takes performing between 50-60 cases to pass the learning curve and reach an expert level and a minimum of 20 cases a year to maintain the surgical skill in VATS, although there is no literature about the time recommended for learning VATS (14).

The learning curve of surgical techniques depends on the volume and frequency with which the technique is performed, since it is important to both acquire and maintain the skill. In addition, the constant supervision of an expert compensates for the inexperience of the trainee surgeon, accelerates learning, increases safety and decreases the risk of complications. In high volume centres, the training to reach an expert level takes place over a shorter period of time (15).

The aim of our study was to analyse the early learning curve of a single surgeon in a high volume training centre and determine the clinical outcomes of uniportal VATS major lung resections, in terms of complications, conversion rate, operating time, length of hospital stay (LOS) and overall postoperative 30 -day mortality.

\section{Methods}

This was a retrospective descriptive study of patients undergoing uniportal VATS major lung resections. All data was collected prospectively from patient medical notes and computerized records. Consent forms were signed by all participating patients. This study was approved by the hospital's Institutional Review Board.

From July 2015 to October 2015, 2,700 patients underwent VATS major lung resection in Shanghai Pulmonary Hospital, Shanghai, China. This included 60 consecutive patients who underwent uniportal VATS lobectomy or segmentectomy by the same surgeon during his early learning curve under supervision. Patients were randomly assigned to different surgical teams in the outpatient clinic, and the choice of surgical approach remained at the attending surgeon's discretion. Preoperative investigations performed routinely included full blood count, serum biochemistry, computed tomography (CT) scans of the chest with $3 \mathrm{D}$ reconstruction, histological diagnostic tests, pulmonary function tests, flexible bronchoscopy and echocardiogram. Positron emission tomography (PET)-CT scans were performed in selected cases. Perioperative histology was carried out in all cases using frozen tissue sections and when an invasive lesion was found, we performed radical lymphadenectomy in all cases.

The previous experience of the trainee in uniportal VATS prior to start the training period was basic and included holding the camera as an assistant, attending uniportal VATS wet-labs, as well as performing all minor procedures through uniportal VATS approach and around 5 major VATS lung resection via a multiport approach under supervision. The mechanism of supervision on the trainee 
was progressive. A very experienced specialist in uniportal VATS (having already performed >5,000 major lung resections by uniportal VATS) was available all the time. At the beginning, he was in the operating room unscrubbed giving advice and observing the surgery. Later on, when the trainee had gained enough experience, the supervisor was either available close to theatres or operating next door, available to give advice. There was no role of the trainee selecting the cases; all cases with inclusion criteria were selected by the supervisor without considering laterality or type of lobectomy and as the trainee's experience and skill progressed, the supervisor provided more complex cases and more challenging surgeries including segmentectomies.

Inclusion criteria for using VATS on patients with lung cancer in our thoracic unit included the following: T1 and $\mathrm{T} 2$ tumour $(<7 \mathrm{~cm}), \mathrm{N} 0$ or $\mathrm{N} 1$ tumour, chest wall involvement of the parietal pleura or ribs, previous thoracic surgery, forced expiratory volume in 1 second (FEV1) of $>40 \%$ and predicted postoperative diffusing capacity of the lungs for carbon monoxide (DLCO) $>40 \%$. Patients with central masses invading hilar structures and/or N2 disease were excluded. Indications for using the uniportal VATS in the early learning curve of a single surgeon included the presence of T1 or T2 tumour $(<7 \mathrm{~cm})$ and N0 status. Exclusion criteria were the following: chest wall involvement, central masses, previous thoracic surgery, adhesions, body mass index (BMI) $>30 \mathrm{~kg} / \mathrm{m}^{2}$, cardiomegaly and enlarged lymph nodes with confirmed N1or N2 disease. Surgery in all cases was performed under general anaesthesia, using single-lung ventilation, by a surgeon and an assistant and after completing the lung resection a systematic radical lymph node dissection was performed.

The following clinical data were collected: age, gender, BMI, weight, smoking habits, pulmonary function tests, associated comorbidities, size and location of the tumour, operating time, intraoperative bleeding, TNM stage, histology type, lymph node involvement, duration of chest drain use, LOS, complications, rate of conversion and surgical mortality. Complications were defined as follows: bleeding not controllable by Uniportal VATS, pneumonia, and prolonged air leak (PAL), defined as any detectable air leak lasting 7 days or more. Surgical mortality was defined as death during hospitalization or within 30 days after the operation.

The patient was given general anaesthesia, with doublelumen endotracheal intubation and placed in a lateral decubitus position. A surgeon and a scrub nurse were positioned in front of the patient, with an assistant standing at the back and a screen placed at the head of the patient. The uniportal approach is performed with an incision of 3 to $4 \mathrm{~cm}$ in the fourth intercostal space for right upper lobectomies and in fifth intercostal space for right middle and lower lobectomies and left side for all resections, introducing the 30-degree video thoracoscope and the dissection instruments for the same wound. The camera should always remain above and the instruments of work by underneath to facilitate bimanual dissection. Vision is anterior and in tunnel, facilitating access and dissection of the hilum and mediastinal stations. In addition, the surgeon has a more ergonomic surgical position (16).

Detailed collection of data was prospectively adhered to throughout the VATS programme. Statistical analysis was performed using SPSS software (version 22; SPSS, Chicago, IL, USA). A descriptive analysis of the variables was carried out. Data was expressed as percentage mean \pm standard deviation, median or range. Analysis with intention to treat, including all conversions and comparison on perioperative outcomes between the first 30 cases and the last 30 cases, was performed and the characteristics and outcome variables were compared using Chi square or Student $\mathrm{T}$ tests depending on the type of variable. Next, linear regression models were carried out in order to identify the minimum number of cases to reach an expert level. In all the tests a significant difference was accepted from a $\mathrm{P}$ value $<0.05$.

\section{Results}

From July 2015 to October 2015, 60 selected consecutive patients ( 35 males and 25 females), with a mean age of $57 \pm 11.4$ years, underwent either uniportal VATS lobectomy or segmentectomy by a single surgeon during his early learning curve in a high volume training centre. Demographic characteristics, comorbidities, pulmonary function tests and outcomes are described in Table 1.

After anatomical resection, systematic lymph node sampling or dissection was performed when frozen section showed malignancy. The number of nodal stations explored in patients with NSCLC $(\mathrm{n}=56)$ was $4.2 \pm 0.8$.

Of the 60 cases, 47 were lobectomies and 13 were segmental resections. Of the lobectomies, right upper lobectomy (RUL) was the most common procedure (42\%). Of the segmental resections, 7 were right sided and 6 left sided. Details of the 60 uniportal VATS major lung resections are described in Table 2.

Table 3 presents the distribution of the final histological 
Table 1 Demographic and clinical data

\begin{tabular}{|c|c|}
\hline Parameter & Values \\
\hline Mean age (years) & $57 \pm 11.4$ \\
\hline \multicolumn{2}{|l|}{ Sex, n (\%) } \\
\hline Male & $35(58.3)$ \\
\hline Female & $25(41.7)$ \\
\hline Mean BMI & $22.3 .0 \pm 0.93$ \\
\hline Mean weight (kg) & $61.8 \pm 9$ \\
\hline \multicolumn{2}{|l|}{ Smoking history, n (\%) } \\
\hline Yes & $33(55.0)$ \\
\hline No & $27(45.0)$ \\
\hline Tuberculosis, n (\%) & $1(1.7)$ \\
\hline Previous cancer, n (\%) & $6(10.0)$ \\
\hline Cardiovascular risk, n (\%) & $7(11.7)$ \\
\hline \multicolumn{2}{|l|}{ Lung function test } \\
\hline FEV1 (litres) & $2.5 \pm 0.5$ \\
\hline FEV1 (\%) & $89.7 \pm 13.0$ \\
\hline FVC (litres) & $3.3 \pm 0.2$ \\
\hline Mean operation time (minutes) & $193 \pm 45.4$ \\
\hline Mean operative blood loss (mL) & $168 \pm 94$ \\
\hline Mean size diameter of the lesions (mm) & $24 \pm 3.3$ \\
\hline Mean chest drain duration (days) & $2.9 \pm 0.9$ \\
\hline Mean LOS (days) & $4.38 \pm 0.4$ \\
\hline Mean lymph node station sampled & $4.2 \pm 0.8$ \\
\hline Conversion to thoracotomy, n (\%) & $1(1.7)$ \\
\hline Conversion to conventional VATS, n (\%) & $0(0)$ \\
\hline Total conversions, $\mathrm{n}(\%)$ & $1(1.7)$ \\
\hline
\end{tabular}

BMI, body mass index; LOS, length of hospital stay; FEV1, forced expiratory volume in 1 second; FVC, forced vital capacity; VATS, video-assisted thoracoscopic surgery.

diagnosis (according to the World Health Organization classification).

There was one case $(1.7 \%)$ in which unexpected intraoperative bleeding, which occurred among the first 30 cases on the left side while attempting a left upper apical segmentectomy. A lymph node was completely attached to the artery and conversion to thoracotomy was required to control the bleeding properly and an angioplasty was performed to complete the resection, owing to lymph node being fused to the left superior pulmonary artery.

Complications encountered in uniportal VATS lobectomy are summarized in Table 4. An analysis with intention to treat, including all perioperative outcomes (first 30 cases $v$ s. last 30 cases), was performed. Surgical re-intervention was never required. There were no readmission, and postoperative 30-day mortality was zero.

Comparing the first 30 cases $v$ s. the last 30 cases, the days of hospital stay were statistically significant $(\mathrm{P}=0.015)$ with an average of 4.73 days in the first 30 cases and 4.03 days in the last 30 cases, and the duration of surgery $(\mathrm{P}<0.001)$ averaged to the first 30 cases of 215.27 and $169.5 \mathrm{~min}$ in the last 30 cases.

The average number of drainage days was slightly higher in the first 30 days, being 3.17 days in the first 30 cases and 2.67 in the last 30 . The bleeding was more abundant in the first 30 days with $170 \mathrm{~mL}$ compared to $165 \mathrm{~mL}$ in the last 30 days. Similarly, complications were more frequent the first 30 cases with $20 \%$, as compared to the last 30 cases $10 \%$. In Figure 1 the values of each variable are described in more detail.

\section{Discussion}

VATS pulmonary resections with radical lymphadenectomy/sampling are in some international centers a routine procedure. However, currently less than $25 \%$ of the largest resections in Europe are performed by VATS (17). Given that VATS is still evolving as a technique, many centers are currently or will soon find themselves facing the early learning curve. Within VATS resections, the uniportal approach is least invasive, but has a longer and more difficult learning curve. While there are currently studies that support the benefits of VATS, there are none that study the learning curve and lymph node dissection (18-21).

The reason that justified the implementation of this technique and triggered the present study was none other than answering the following questions:

Is VATS surgery during the safe, effective learning curve and meets international quality recommendations?

There are few papers in literature describing the early learning curve of major pulmonary resections by VATS in Thoracic Surgery, and even fewer regarding experience with a uniportal approach.

Ferguson et al. reports that when a surgeon under training is closely supervised by a surgeon with extensive VATS experience, safety, 30-day survival, blood loss intra- 
Table 2 Complete 60 uniportal VATS major lung resections, including 47 lobectomies and 13 segmentectomies

\begin{tabular}{|c|c|c|c|c|}
\hline Type of surgery & Right side & Frequency (n) & Left side & Frequency $(\mathrm{n})$ \\
\hline \multirow{2}{*}{ Lobectomy (n=47) } & RML & 2 & LLL & 4 \\
\hline & RLL & 4 & & \\
\hline \multirow[t]{3}{*}{ Segmental resections $(n=13)$} & Apical (S1) & 3 & Trisegmentectomy $(\mathrm{S} 1+\mathrm{S} 2+\mathrm{S} 3)$ & 3 \\
\hline & Apico-posterior (S1+S2) & 1 & & \\
\hline & Superior (S6) & 2 & & \\
\hline
\end{tabular}

VATS, video-assisted thoracoscopic surgery.

Table 3 Histological types of surgically removed specimens in 60 patients with primary NSCLC

\begin{tabular}{lc}
\hline Histological types $(\mathrm{N}=60)$ & Data, $\mathrm{n}(\%)$ \\
\hline Malignant, $56(93.3 \%)$ & $9(15.0)$ \\
Lung cancer & $11(18.3)$ \\
Adenocarcinoma in situ & $28(46.7)$ \\
Microinvasive adenocarcinoma & $8(13.3)$ \\
Adenocarcinoma & $1(1.7)$ \\
Squamous cell carcinoma & $2(3.3)$ \\
Benign, 4 (6.7\%) & $1(1.7)$ \\
Atypical adenomatous hyperplasia &
\end{tabular}

NSCLC, non-small cell lung cancer.

Table 4 Comparison on perioperative outcomes (first 30 cases vs. last 30 cases)

\begin{tabular}{lccc}
\hline Parameter & First 30 cases $(\mathrm{N}=30)$ & Last 30 cases $(\mathrm{N}=30)$ & $\mathrm{P}$ value \\
\hline Mean age (years) & $56.4 \pm 2.3$ & $58.7 \pm 5.3$ & 0.96 \\
Chest drain duration (days) & $3.1 \pm 1.21$ & $2.6 \pm 0.6$ & 0.07 \\
Days of hospital stay (days) & $4.7 \pm 1.2$ & $165.1 \pm 109$ & 0.015 \\
Intraoperative bleeding (mL) & $170.8 \pm 78$ & $169.5 \pm 42$ & 0.02 \\
Duration of surgery (mins) & $215.2 \pm 36$ & 0 & 0.001 \\
Conversions thoracotomy & $1(1.7 \%)$ & $3(10 \%)$ & 0 \\
Complications & $6(20 \%)$ & 2 & 0.42 \\
PAL & 4 & 0 \\
Pneumonia & 2 & 1 \\
Wound infection & 0 & & 0 \\
\hline
\end{tabular}



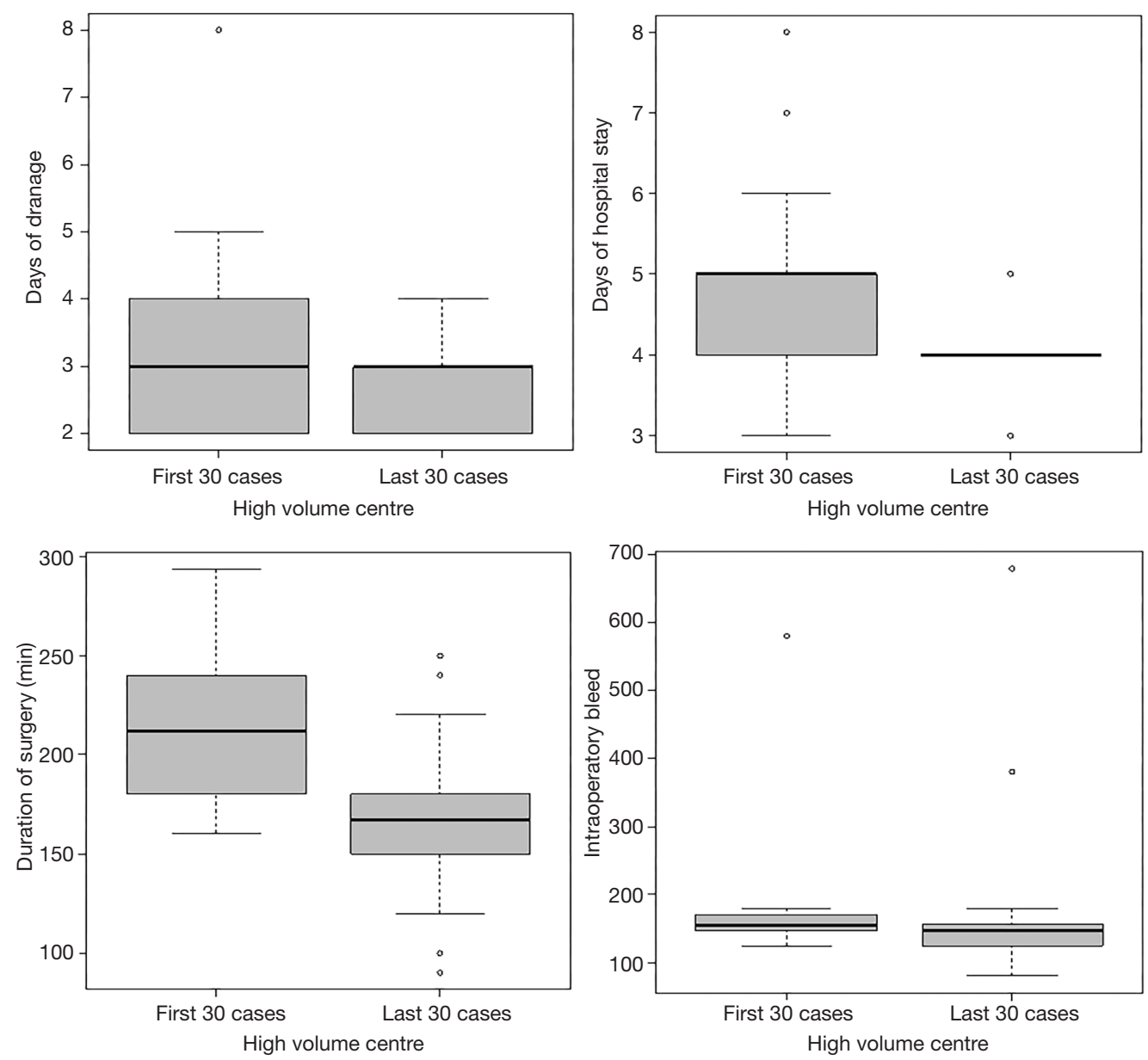

Figure 1 Comparison on perioperative outcomes (first 30 cases vs. last 30 cases).

operatively and complications are similar, reporting only a longer surgical time (22).

Some studies describe that after their first 50 cases of VATS learning curve in their own institution, the surgical times, blood loss during surgery and mortality improve considerably $(23,24)$.

Other authors recommend that the surgeon under training spend a long and significant period of training with a surgeon very experienced in VATS surgery to be able to acquire the skill and confidence to start their own practice, after which they can return to their previous workplace to recreate and follow their own VATS programme. They also advocate frequent visits to their training center to hone their skills and answer the inevitable doubts and questions that arise as more experience is gained and more complex cases are encountered (25).

By analyzing the learning curve we have learned that there is no significant difference between a conventional
VATS curve described in the literature and a uniportal VATS curve, of which there is sparse reference. The difference arises when carrying out the learning curve in a high volume training center with an active tutelage of a surgeon since complications, morbidity and mortality decrease, as well as the number of bleeding and errors due to anatomical confusion.

Despite starting a new technique, 30-day patient survival is not compromised during the learning curve in any of the minimally invasive centers.

After analyzing the results it is highly recommended to perform the learning curve in a high volume training center supervised by an expert since the learning curve is faster, the lymph node dissection is safer and more efficient, an expert level is reached sooner and more complex cases can be performed. In the Figure 2, we can observe the operation time decreasing in time when increasing procedural experience, showing that a good level in a high volume 


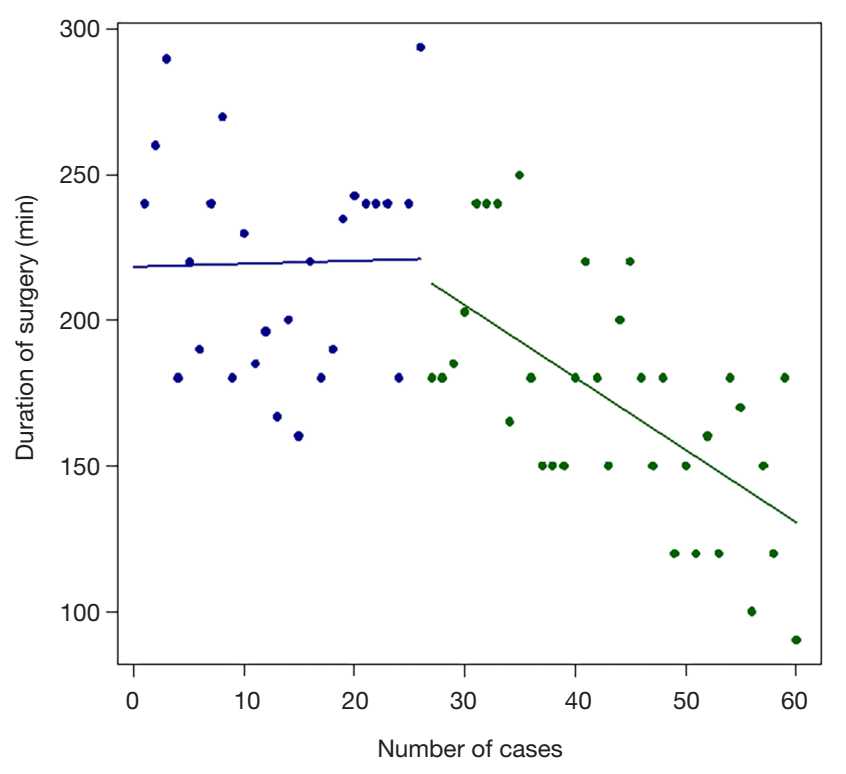

Liner model that adjusts the first 26 cases: [Duration of surgery $(\mathrm{min})]=218.21+0.10^{*}$ (Number of cases) Linear model that adjusts the last 34 cases: [Duration of surgery $(\min )]=279.50-2.48^{\star}$ (Number of cases)

Figure 2 Number of cases to reach expert level according to the duration of surgery.

center can be reached in the case number 26 to 30 .

In conclusion, based on our findings, we recommend learning Uniportal VATS or any other VATS approach in a high volume training centre where surgeons can reach the expert level faster (60 cases in 4 months) and perform more complex resections in less time. Further studies are necessary to validate the applicability, by comparing other experiences of more surgeons in a high volume training centre and also comparing the outcomes of the learning curve of a surgeon trained in a high volume training centre with those of a surgeon trained in the traditional way (doing courses, watching videos, visiting centre for a short time and supervision).

\section{Acknowledgements}

None.

\section{Footnote}

Conflicts of Interest: The authors have no conflicts of interest to declare.

Ethical Statement: This study was approved by the hospital's Institutional Review Board. Consent forms were signed by all participating patients.

\section{References}

1. Yamamoto H, Okada M, Takada M, et al. Video-assisted thoracic surgery through a single skin incision. Arch Surg 1998;133:145-7.

2. Rocco G, Martin-Ucar A, Passera E. Uniportal VATS wedge pulmonary resections. Ann Thorac Surg 2004;77:726-8.

3. Rocco G. Single port video-assisted thoracic surgery (uniportal) in the routine general thoracic surgery practice. Op Tach Stcvs 2009;14: 326-35.

4. Rocco G, Martucci N, La Manna C, et al. Ten-year experience on 644 patients undergoing single-port (uniportal) video-assisted thoracoscopic surgery. Ann Thorac Surg 2013;96:434-8.

5. Gonzalez-Rivas D, de la Torre M, Fernandez R, et al. Single-port video-assisted thoracoscopic left upper lobectomy. Interact Cardiovasc Thorac Surg 2011;13:539-41.

6. Ismail $M$, Helmig $M$, Swierzy $M$, et al. Uniportal VATS: the first German experience. J Thorac Dis 2014;6:S650-5.

7. Aragón J, Pérez Méndez I. From open surgery to uniportal VATS: asturias experience. J Thorac Dis 2014;6:S644-9.

8. Gonzalez-Rivas D, de la Torre M, Fernandez R, et al. Video: Single-incision video-assisted thoracoscopic right pneumonectomy. Surg Endosc 2012;26:2078-9.

9. Gonzalez-Rivas D, Fieira E, Mendez L, et al. Single-port video-assisted thoracoscopic anatomic segmentectomy and right upper lobectomy. Eur J Cardiothorac Surg 2012;42:e169-71.

10. Gonzalez-Rivas D, Fernandez R, Fieira E, et al. Uniportal video-assisted thoracoscopic bronchial sleeve lobectomy: first report. J Thorac Cardiovasc Surg 2013;145:1676-7.

11. Gonzalez-Rivas D, Delgado M, Fieira E, et al. Single-port video-assisted thoracoscopic lobectomy with pulmonary artery reconstruction. Interact Cardiovasc Thorac Surg 2013;17:889-91.

12. Hernandez-Arenas LA, Lin L, Yang Y, et al. Initial experience in uniportal subxiphoid video-assisted thoracoscopic surgery for major lung resections. Eur J Cardiothorac Surg 2016;50:1060-6.

13. Harris CG, James RS, Tian DH, et al. Systematic review 
and meta-analysis of uniportal versus multiportal videoassisted thoracoscopic lobectomy for lung cancer. Ann Cardiothorac Surg 2016;5:76-84.

14. Yan TD, Cao C, D'Amico TA, et al. Video-assisted thoracoscopic surgery lobectomy at 20 years: a consensus statement. Eur J Cardiothorac Surg 2014;45:633-9.

15. Hernandez-Arenas LA, Guido W, Jiang L. Learning curve and subxiphoid lung resections most common technical issues. J Vis Surg 2016;2:117.

16. Bertolaccini L, Viti A, Terzi A, et al. Geometric and ergonomic characteristics of the uniportal video-assisted thoracoscopic surgery (VATS) approach. Ann Cardiothorac Surg 2016;5:118-22.

17. Nagahiro I, Andou A, Aoe M, et al. Pulmonary function, postoperative pain, and serum cytokine level after lobectomy: a comparison of VATS and conventional procedure. Ann Thorac Surg 2001;72:362-5.

18. American Cancer Society. Cancer Prevention \& Early Detection Facts \& Figures 2015-2016. Atlanta: GACS, 2015.

19. Downey RJ, Martini N, Rusch VW, et al. Extent of chest wall invasion and survival in patients with lung cancer. Ann
Thorac Surg 1999;68:188-93.

20. de Perrot M, Fadel E, Mercier O, et al. Long-term results after carinal resection for carcinoma: does the benefit warrant the risk? J Thorac Cardiovasc Surg 2006;131:81-9.

21. Battafarano RJ, Meyers BF, Guthrie TJ, et al. Surgical resection of multifocal non-small cell lung cancer is associated with prolonged survival. Ann Thorac Surg 2002;74:988-93; discussion 993-4.

22. Ferguson J, Walker W. Developing a VATS lobectomy programme--can VATS lobectomy be taught? Eur J Cardiothorac Surg 2006;29:806-9.

23. Zhao H, Bu L, Yang F, et al. Video-assisted thoracoscopic surgery lobectomy for lung cancer: the learning curve. World J Surg 2010;34:2368-72.

24. Belgers EH, Siebenga J, Bosch AM, et al. Complete videoassisted thoracoscopic surgery lobectomy and its learning curve. A single center study introducing the technique in The Netherlands. Interact Cardiovasc Thorac Surg 2010;10:176-80.

25. Dunning J, Walker WS. How to set up a VATS lobectomy program. Ann Cardiothorac Surg 2012;1:43-6.
Cite this article as: Hernandez-Arenas LA, Lin L, Purmessur RD, Zhou Y, Jiang G, Zhu Y. Uniportal video-assisted thoracoscopic early learning curve for major lung resections in a high volume training center. J Thorac Dis 2018;10(Suppl 31):S3670-S3677. doi: 10.21037/jtd.2018.04.16 